OPEN ACCESS

Edited by:

Lei Deng,

Central South University, China

Reviewed by:

Mingzhi Liao,

Northwest A\&F University Apple

Research Center, China

Juan Wang

Inner Mongolia University, China

${ }^{*}$ Correspondence: Ying Jiang

jiangying1222@163.com

Donghua Wang wangdonghua7885@126.com

lijhlifj@126.com

Specialty section:

This article was submitted to

Molecular Medicine,

a section of the journal

Frontiers in Cell and Developmental

Biology

Received: 19 June 2020 Accepted: 14 August 2020

Published: 31 August 2020

Citation:

Jiang $Y$, Song $H$, Jiang $L$, Qiao $Y$,

Yang D, Wang D and Li J (2020) Silybin Prevents Prostate Cancer by Inhibited the ALDH1A1 Expression in the Retinol Metabolism Pathway.

Front. Cell Dev. Biol. 8:574394.

doi: 10.3389/fcell.2020.574394

\section{Silybin Prevents Prostate Cancer by Inhibited the ALDH1A1 Expression in the Retinol Metabolism Pathway}

\author{
Ying Jiang ${ }^{1 *}$, Hanbing Song ${ }^{2}$, Ling Jiang ${ }^{1}$, Yu Qiao ${ }^{1}$, Dan Yang ${ }^{1}$, Donghua Wang ${ }^{3 *}$ and \\ Ji Li ${ }^{1 *}$ \\ ${ }^{1}$ College of Basic Medicine, Heilongjiang University of Chinese Medicine, Harbin, China, ${ }^{2}$ The First Affiliated Hospital, \\ Heilongjiang University of Chinese Medicine, Harbin, China, ${ }^{3}$ Department of General Surgery, General Hospital \\ of Heilongjiang Province Land Reclamation Bureau, Harbin, China
}

Background: Silybin was known to exert inhibition in prostate cancer, but the underlying mechanism remained largely unknown. This study was designed to find out the potential target of Silybin on prostate cancer and explore the relative mechanisms.

Methods: Firstly, we screened the possible targets of Silybin through the PubChem database and Subpathway - GM. Then DU145 cells were transferred to investigate the correction about related targets, magnetic bead sorting and flow cytometry were used to sort and identify the cells. Proliferation, migration and invasion ability of DU145 cells were detected by MTT assay, Transwell assay, plate clonality and sphere formation assay. BALB/c nude mice were constructed models with implanted sarcoma and measured the tumor volume every 5 days as wells tumor weight. The levels of proteins were detected by Western blot and immunocytochemistry. RT-PCR was selected to test the expression of protein's mRNA.

Results: It was screened out the ALDH1A1 was highly correlated with subpathways of the Silybin risk metabolic pathway. And ALDH1A1 expression was positively correlated RAR $\alpha$ with Ets1 by interfering with the ALDH1A1 gene. Importantly, ALDH1A1(+) cells showed proliferation, migration and invasion ability. In addition, it showed that Silybin exerted the inhibition on prostate cells by suppressed the proliferation, migration and invasion ability of cells in vitro experiment. Silybin also reduced the tumor volume and weight. And Silybin displayed obviously reduced the proteins and mRNA of ALDH1A1, RAR $\alpha$, Ets1 and MMP9 expressions.

Conclusion: Our results indicated that Silybin showed inhibition of prostate cancer and the mechanism was involving with downregulating ALDH1A1 expression, thereby inhibiting the activation of RAR $\alpha$ and preventing the activation of Ets 1 to inhibit the growth and invasion of prostate cancer.

Keywords: prostate cancer, ALDH1A1, silybin, metabolism pathway, biomarker 


\section{INTRODUCTION}

Prostate cancer is one of the malignant tumors that endanger the health of men around the world and the incidence is getting more common in recent years (Arcangeli et al., 2012; Kimura and Egawa, 2018; Lin et al., 2019; Qu et al., 2019; Zeng et al., 2019; Zhou et al., 2019). At present, the common treatment options were radical prostatectomy, chemotherapeutic drugs, anti-androgenic therapies, immunotherapy and so on (Ahmad et al., 2017; Gamat and McNeel, 2017; Lawrence et al., 2018; Liao et al., 2018; Liu et al., 2018; Tang et al., 2018; Ji et al., 2019; Han et al., 2020). However, risk factors of these treatments, such as permanent urinary incontinence, peripheral neuropathy and high cost, limited the applications (Green et al., 2015). Therefore, it was of great theoretical and practical significance to find effective treatment for prostate cancer.

Silybin, a mixture of flavonolignan and flavonoid polyphenolic compounds extractable from milk thistle seeds, had high antioxidant and anticancer properties, which caused broadspectrum efficacy against cancer (Jahanafrooz et al., 2018; Di Costanzo and Angelico, 2019). Actually, Silybin had been widely investigated for anti-cancer efficacy in a broad range of cancers models such as cervical cancer, liver cancer and prostate cancer especially (Varghese et al., 2005; Ting et al., 2013). Some research findings revealed that Silybin could inhibit the secretion of angiogenic factors and targeting the responses of endothelial angiogenic factors (motility, proliferation, survival, and differentiation) effectively for the treatment of prostate cancer cells (Singh et al., 2008). Evidence suggested that ALDH1A1 was significantly associated with prostate cancer risk and which could be a prostate cancer stem cells-related marker (Li et al., 2010). Furthermore, Silybin could downregulated the expression of ALDH1A1 to alleviate the process of neck squamous cell carcinomas (Hellsten et al., 2011). And it had also been indicated that Silybin could significantly induced the expression of matrix metalloproteinases (MMPs) to prevent the tumor metastasis in vitro and vivo studies (Deep and Agarwal, 2010). Although Silybin could inhibit prostate cancer cell proliferation, its effect on stem cell-like cells from prostate cancer is unclear. Presuming finding the potential targets on prostate stem cancer would have great prospect.

Recently, it had become one of the most important focuses to screen drug targets by bioinformatics method which was beneficial to improve the safety and reliability of drug application (Gao et al., 2018; Lai et al., 2018; Cheng et al., 2019; Feng, 2019; Su et al., 2019). For example, Huang et al. (2017) identified some hub genes of prostate cancer through an integrated bioinformatics approach and Foj and Filella (2019) identified of potential miRNAs biomarkers for high-grade prostate cancer by integrated bioinformatics analysis. As a matter of fact, the development of bioinformatics technology had not only made great achievements in disease mechanism research and diagnosis and treatment (Han et al., 2018; Zhang et al., 2020), but also provided new concepts and means in the way of finding new drug action targets and the preparation process, which greatly promoted the pace of medical research and development from both theoretical and technical aspects (Du et al., 2018; Di et al., 2019; Sun et al., 2019; Wu et al., 2019; Cheng et al., 2020).

In this study, we firstly obtained potential targets of Silybin for prostate cancer by bioinformatics method and then confirmed the related pathology on proliferation, migration and invasion of prostate stem cancer. And we further explored the mechanism of Silybin in inhibiting prostate cancer by the mechanism in vitro and vivo.

\section{MATERIALS AND METHODS}

\section{Target-Based Screening by Bioinformatics Analysis}

We found the possible targets of Silybin through the PubChem database $^{1}$, and obtained 26 active targets and then screen for the highly correlated target by inputting the NCBI-Gene ID of each gene into Subpathway - GM² (Li et al., 2013).

\section{Chemicals and Regents}

Silybin (HY-13748) was bought from Medchemexpress (NJ, United States). RPMI-1640 (31800-014) was purchased from Gibco (Shanghai, China). Anti-Biotin MicroBeads (130-105-637) was obtained from Miltenyi Biotechnology Co. Ltd. (Kölner, German). Biotin-labeled ALDH1A1 antibody (NBP2-54422B) was got from Novus Biologicals (CO, United States). Polyclonal antibodies of ALDH1A1 (WL02762), Ets1 (WL02523), MMP9 (WL03096) were bought from Wanlei Biotechnology Co. Ltd. (Wuhan, China) and RAR $\alpha$ (10331-1-AP) were bought from proteintech group, Inc. (Wuhan, China). RNase inhibitor (RP5602), $2 \times$ Power Taq PCR MasterMix (PR1702) and Super $\mathrm{m}$-mlv reverse transcriptase (PR6502) were got from BioTeck Biotechnology Co. Ltd (Beijing, China). Retinoic Acid (ATRA, R106320) was bought from Aladdin (Shanghai, China).

\section{In vitro Experiment}

\section{Cell Treatment and Transfection}

DU145 was obtained from bought from Wanlei Biotechnology Co. Ltd. (Wuhan, China). DU-145 cells were cultured with RIMI-1640 containing $10 \% \mathrm{FBS}$ at $37^{\circ} \mathrm{C}$ and $5 \% \mathrm{CO}_{2}$ in the incubator. Using trypsin to digest DU-145 cells in logarithmic growth stage and disperse them into a cell suspension, and dilute the cell density to $2 \times 10^{5} / \mathrm{mL}$. The cells were seeded in $96-$ well plates at the density of $2 \times 10^{4} /$ well. Lipofectamine ${ }^{\mathrm{TM}} 2000$ was transfected randomly into DU-145 cells. The experience consisted of five groups: Untranslated group, negative matched control transfection group and transfection group (siRNA-1, siRNA-2, siRNA-3) (Table 1). According to the ALDH1A1 gene sequence to determine the start site of gene transcription, and the specific interference sites were listed in the Supplementary Figure S1. Successful transfections were determined using quantitative RT-PCR or the western blot assay. ALDH1A1+ cells were treated with DMSO, Silybin $(50,100150,200 \mu \mathrm{M})$ and siRNA-ALDH1A1 for $48 \mathrm{~h}$.

\footnotetext{
${ }^{1}$ http://pubchem.ncbi.nlm.nih.gov/

${ }^{2} \mathrm{https} / /$ github.com/chunquanlipathway/iSubpathwayMiner
} 
TABLE 1 | siRNA sequence of ALDH1A1.

\section{Name}

ALDH1A1-homo-591

ALDH1A1-homo-924

ALDH1A1-homo-1288

Negative Control
Sequence

GGCUGAUUUAAUCGAAAGATTUCUUUCGAUUAAAUCAGCCTT

CCACGUGGCAUCUUUAAUATTUAUUAAAGAUGCCACGUGGTT GAGCGGGCUAAGAAGUAUATTUAUACUUCUUAGCCCGCUCTT UUCUCCGAACGUGUCACGUTTACGUGACACGUUCGGAGAATT

\section{Magnetic Bead Cell Sorting}

Cells with a cell density of $1 \times 10^{8} / \mathrm{mL}$ were placed in a test tube and incubated in biotin-labeled ALDH1A1 antibody for $5 \mathrm{~min}$. Centrifuged for $10 \mathrm{~min}$ at $300 \mathrm{~g}$ and washed twice with PBS. After obtaining the reclosed cells, the MS separation column was placed in the magnetic field of the MACS separator and rinsed with $500 \mu \mathrm{L}$ Buffer. The $500 \mu \mathrm{L}$ Buffer cell suspension was passed through the separation column and the separation column was rinsed with 500 Buffer collected the effluent to get ALDH1A1cells. ALDH1A1+ cells were removed from the separator and flushed with $1 \mathrm{~mL}$ Buffer to collect the outflow.

\section{MTT Assay}

After DU145, ALDH1A1+, ALDH1A1 - cells adherence for 0, 24, 48, 72, $96 \mathrm{~h}$ and ALDH1A1+ cells treatment with drugs for $48 \mathrm{~h}$, the culture medium was replaced with $0.5 \mathrm{mg} / \mathrm{mL}$ MTT solution in fresh medium and incubated for another $4 \mathrm{~h}$. The supernatant was discarded while the formazan was resolved in $100 \mu \mathrm{L}$ DMSO. Optical density (OD) values were read at a wavelength of $570 \mathrm{~nm}$ by Microplate reader replaces (ELX-800, BIOTEK, United States). All results were repeated for three times.

\section{Quantification of ALDH1A1+ Cells by Flow Cytometry} The cells were washed twice with PBS and collected by centrifugation at $300 \mathrm{~g}$ for $5 \mathrm{~min}$. Hundred $\mu \mathrm{L}$ PBS resuscitation cells containing ALDH1A1 antibody were added and incubated for $30 \mathrm{~min}$ in the dark. And cells were collected by centrifugation at $300 \mathrm{~g}$ for $5 \mathrm{~min}$, the supernatant was discarded, washed with PBS for two times, centrifuged at $300 \mathrm{~g}$ for $5 \mathrm{~min}$, the supernatant was discarded, $500 \mu \mathrm{L}$ staining buffer was added to each tube of cell samples. Finally, cells were stored in ice bath to avoid light, and then enumerated by flow cytometry.

\section{Migration Assay}

Cells were seeded in 24-well plate at the density of $1 \times 10^{3} /$ well at $37^{\circ} \mathrm{C}$ and $5 \% \mathrm{CO}_{2}$ in the incubator for $24 \mathrm{~h}$. Wash the Transwell chamber twice with PBS. Fix $4 \%$ paraformaldehyde for $20 \mathrm{~min}$ at room temperature, dye $0.5 \%$ crystal violet solution for $5 \mathrm{~min}$, rinse with distilled water. The number of cells migrating to the subcellular membrane was counted under an inverted microscope $(200 \times)$. Five fields were selected for cell count for each well, and the mean number was taken as the cell number of migration of the well.

\section{Invasion Assay}

The Transwell chamber was placed into a 24-well plate, coated with $40 \mu \mathrm{L}$ Matrigel prediluted onto the compartment membrane and the gel was placed in an incubator at $37^{\circ} \mathrm{C}$ for $2 \mathrm{~h}$ to solidify. The coated Transwell chamber was placed into a 24-well plate, and $800 \mu \mathrm{L}$ of culture medium containing $30 \%$ FBS was added to the lower chamber. Two hundred $\mu \mathrm{L}$ cell suspensions were added to the upper chamber, and the cell Numbers were $1 \times 10^{4} /$ well at $37^{\circ} \mathrm{C}$ and $5 \% \mathrm{CO}_{2}$ in the incubator for $24 \mathrm{~h}$. Transwell chamber was washed twice with PBS and fixed $4 \%$ paraformaldehyde for $20 \mathrm{~min}$ at room temperature, dye $0.5 \%$ crystal violet solution for $5 \mathrm{~min}$, rinse with distilled water. The number of cells invading to the subcellular membrane was counted under an inverted microscope $(200 \times)$. Five fields were selected for cell count for each well, and the mean number was taken as the cell number of invasion of the well.

\section{Plate Clonality Assays}

Cells were plated in plates at the density of $100 /$ plate and cultured at $37^{\circ} \mathrm{C}$ with $5 \% \mathrm{CO}_{2}$ for 2 weeks. After colony formation, the culture medium was discarded, washed with PBS for two times, fixed with paraformaldehyde for $20 \mathrm{~min}$, stained with Reggie's complex dye for $5 \mathrm{~min}$, and washed with PBS for excess Reggie's complex dye.

\section{Sphere Formation Assay}

The cells were inoculated in 500/well in 6-well ultra-low adsorption cell culture plate with serum-free rpmi-1640 medium (adding $20 \mathrm{ng} / \mathrm{mL}$ EGF, $10 \mathrm{ng} / \mathrm{mL}$ bFGF, $5 \mathrm{~nm}$ glutamate, $1 \%$ cyn-streptomycin, $0.2 \% \mathrm{BSA}, 1 \times \mathrm{B} 27$ without vitamin $\mathrm{A}$, $1 \times$ insulin iron-selenium transfer protein). The culture medium was changed every 3-4 days, and the formation of cell pellets was observed for 2-3 weeks.

\section{Immunocytochemistry}

Expression of ALDH1A1 was assessed by immunocytochemistry. Briefly, the cells were incubated with $0.1 \%$ TritonX-100 for $20 \mathrm{~min}$. After incubated in 3\% hydrogen peroxide for $15 \mathrm{~min}$, sections were blocked with serum for $15 \mathrm{~min}$ at room temperature. And then incubated with rabbit anti-goat (1:100) antibodies overnight at $4^{\circ} \mathrm{C}$. After that, cells were incubated with appropriate secondary antibodies, labeled with horseradish peroxidase and then stained with DAB \& Hematoxylin.

\section{Animals and Treatment}

Six-week-old female BALB/c nude mice were bought from Beijing HFK Bioscience Co. Ltd. [SCXK(Jing)2014-0004]. They were given free way to get food and water with humidity of $50 \pm 10 \%$ at $23 \pm 2{ }^{\circ} \mathrm{C}$ or a $12 \mathrm{~h} \mathrm{light/dark}$ cycle. The mice were randomly divided into groups: Control group and silybin treatment group. A logarithmic growth phase of ALDH1A1 + prostate cancer cells and Silybin-treated $(200 \mu \mathrm{M})$ ALDH1A1+ prostate cancer cells were selected to prepare a cell suspension. $0.2 \mathrm{~mL}$ cells containing $1 \times 10^{6}$ cells were taken and 
inoculated into the right armpit subcutaneously of mice. After waiting for the formation of the tumor in nude mice, record the tumor volume every 5 days. Mice were sacrificed after 5 weeks, and tumor tissues were collected for subsequent experiments.

\section{Real-Time PCR Analysis}

The expression of ALDH1A1 mRNA was detected by Real-time PCR $24 \mathrm{~h}$ after transfection. Firstly, total RNA was extracted by Trizol method. Then, RNA concentrations in each sample were determined using an ultraviolet spectrophotometer (NANO 2000, Thermo, United States). The RNA samples obtained above were reverse-transcribed to obtain the $20 \mu \mathrm{L}$ cDNA according to the manufacturer's specifications. Fluorescence quantitative analysis was carried out with fluorescence quantifier (Exicycler 96, BIONEER, United States). The primers were shown as Table 2. The $2^{-\Delta \Delta \mathrm{Ct}}$ method to calculate the relative expression of the purpose gene mRNA.

\section{Western Blotting}

Western Blot was used to detect to ALDH1A1, RAR $\alpha$, Ets1 and MMP9. The proteins were extracted with radio immunoprecipitation (RIPA) buffer and the concentration was quantitated by BCA assay. The total protein $(100 \mu \mathrm{g})$ were separated on $10 \%$ resolving SDS - PAGE gel and 5\% stacking gel, and transferred to PVDF membrane. Next, membranes were blocked with $5 \%$ skim milk in Tris - buffered saline containing $0.1 \%$ Tween - 20 (TBST) for $1 \mathrm{~h}$, incubated with the primary antibody overnight at $4^{\circ} \mathrm{C}$, and then incubated with horseradish peroxidase - conjugated secondary antibody after washing with TBST. Membranes were visualized using an enhanced chemiluminescence (ECL) detection system. The density of each band was estimated using the Image Lab software. All target proteins were normalized against the loading control $\beta$-actin.

\section{Statistical Analysis}

Each experiment was performed at least three times, and all results were presented as means \pm SD. Results were analyzed with IBM SPSS Statistics 19.0 (SPSS Inc., NY, United States). Significant differences were determined by a Student's $t$-test and one-way analysis of variance (ANOVA) $\left({ }^{*} P<0.05\right.$ or $\left.{ }^{* *} P<0.01\right)$.

\section{RESULTS}

\section{Silybin Target Screening}

Firstly, we used bioinformatics methods to analyze of the new target of Silybin. There were 26 active targets which are possible targets of Silybin, which obtained from PubChem database. Then the NCBI-Gene ID for each gene was entered into the Subpathway-GM system, and a sub-pathway of the retinol metabolism was identified. The result shown that the prostate cancer stem cell marker ALDH1A1 (EC 1.2.1.36) (Red nodes in Figure 1) are key enzyme in the sub-pathway.

\section{The Correlation Among ALDH1A1 and RAR $\alpha$ and Ets1 Expression in Prostate Cancer Cells}

To characterize the correlation of ALDH1A1, RAR $\alpha$ and Est1, we measured the expressions of them after DU145 cells transfection by Western blotting and RT-PCR. The results showed that the expression of ALDH1A1 proteins and mRNA in transfection groups (siRNA-1, siRNA-2, siRNA-3) were significantly reduced compared with untreated group $(P<0.01)$ (Figures 2A,D,G). In the meanwhile, the productions of RAR $\alpha$ and Est1 in transfections were also lower than untreated group which were transfected by siRNA-1 and siRNA-2 $(P<0.01)$, but it was no statistical difference by siRNA-3 $(P>0.05)$ (Figures 2B,C,E,F). Therefore, siRNA-1 was chosen to use in the subsequent experiments. The results indicated ALDH1A1 expression was positively correlated RAR $\alpha$ with Ets1.

\section{ALDH1A1(+) (-) Cell Sorting and Biological Function}

Flow cytometry was used to sort and identify the ALDH1A1(+) cells. It was showed that cells containing ALDH1A1 gene were $4.2 \%$ in DU145 cells, $97.0 \%$ in ALDH1A1(+) cells and $0.8 \%$ in ALDH1A1(-) cells, respectively (Figure 3A). Then MTT assay results showed that ALDH1A1(+) cells group exhibited higher cell proliferative capacity than DU145 cells, but cell proliferative capacity of ALDH1A1(-) cells were obviously reduced compared to DU145 cells, after incubation 24-96 h (Figure 3B). And we also detected the proliferation ability by plate clonality which indicated that colony forming efficiency was higher in ALDH1A1 $(+)$ cells, but lower in ALDH1A1 $(-)$ cells, than in DU145 cells (Figures 3C,J). The results of RT-PCR verified that ALDH1A1 mRNA expression level in ALDH1A1(+) cells were significantly increased while was significantly reduced in ALDH1A1(-) cells compared to DU145 Cells (Figure 3D). After Transwell assay, the abilities of migration and invasion in ALDH1A1 $(+)$ cells were strengthened, whereas the abilities in ALDH1A1(-) cells were weakened, in comparison to DU145 cells (Figures 3E,F,I). Furthermore, we examined the protein of ALDH1A1 by immunocytochemistry and it was showed that the protein of ALDH1A1 was largely expression in ALDH1A1(+) cells but little expression in either ALDH1A1(-) cells or DU145 cells (Figure $\mathbf{3 H}$ ). As is shown in Figure 3G, the number of ALDH1A1 $(+)$ cells continued to increase until the cells formed small spheres and many formed dense spheres, but no obvious globular formation was observed in $\operatorname{ALDH} 1 \mathrm{Al}(-)$ cells after 2-3 weeks of cells inoculation.

\section{The Effect of Silybin on ALDH1A1(+) Cells}

Having known that ALDH1A1(+) cells had high ability of proliferation, migration and invasion based on the above experiments. We further investigated whether Silybin inhibited prostate cancer stem cells growth in vitro. MTT assay showed that the cells' ability to proliferate was inhibited after treated with different concentrations (100, 150, $200 \mu \mathrm{M})$ for $24 \mathrm{~h}$ (Figure 4A). And Silybin also inhibited cell migration and invasion according 


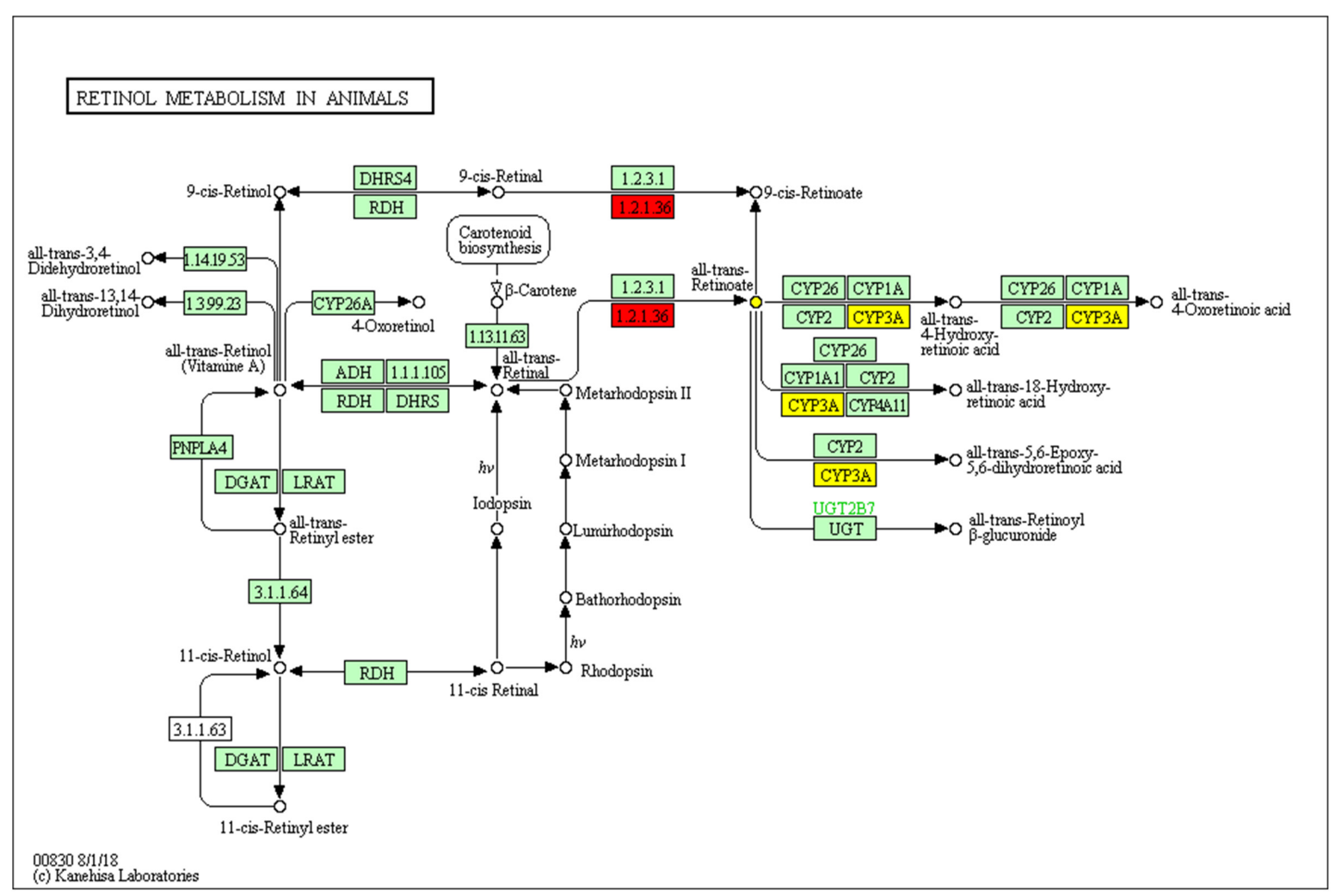

FIGURE 1 | The local area (subpathway) of retinol metabolism pathway is highly correlated with ALDH1A1. The rectangles represent the enzymes and the circles represent compounds. The enzymes encoded by ALDH1A1 are marked with red. The red and yellow nodes represent the subpathway of the pathway.

to the Transwell assay (Figures $4 \mathbf{B}-\mathbf{E}$ ). Then, the expressions of ALDH1A1, RAR $\alpha$, Ets1 were detected by Western blotting and the results showed that Silybin at the concentrations of 50-200 $\mu \mathrm{M}$ could reduce the productions of ALDH1A1, RAR $\alpha$ and Ets1 (Figures 4F-M). Collectively, these results indicated that Silybin could inhibit prostate cancer by downregulating the expressions of ALDH1A1, RAR $\alpha$ and Ets1 in vitro.

To further verify the antitumous role in prostate cancer, the mice models with implanted sarcoma were constructed (Figure 5A). And we recorded the tumor volume every 5 days after the tumor formed. The results showed that tumor volume in control group were greater than Silybin group after day 10 and the result was maintained until the mice sacrifice (Figures 5B,C). Also, tumor weight in control group was heavier than Silybin group (Figure 5D). According to the Western bolt results, the Silybin group exhibited lower expressions of ALDH1A1, Ets1, RAR $\alpha$ and MMP9 than in control group (Figures 5E-L). Concluded from the above experiments, Silybin could reduce the levels of ALDH1A1, Ets1, RAR $\alpha$ and MMP9 to inhibit the tumor growth in vivo.

\section{DISCUSSION}

In this study, we firstly screened out the ALDH1A1, which was highly correlated with subpathways of the Silybin risk metabolic pathway by bioinformatics method and then investigated the effect of ALDH1A1 expression on proliferation, migration and invasion of prostate stem cancer. And we further explored the mechanism of Silybin in inhibiting prostate cancer in vitro and vivo.

It wound greatly advance our understanding of tumor biology of prostate to develop the specific makers of prostate cancer. Recent studies have revealed that aldehyde dehydrogenase1A1 (ALDH1A1), an aldehyde oxidase that can degrade toxic aldehydes in cells to maintain a stable environment in the cell, was a marker for malignant prostate stem cells and predictor of prostate cancer patients' outcome (Yoshida et al., 1992; Li et al., 2010). A clinical research indicated that the positive expression of ALDH1A1 in 163 cases of prostate cancer tissue was significantly increased in secretory carcinoma epithelioid cells and neuroendocrine tumor cells and the cells with positive expression had strong clonality and tumorigenicity (Li et al., 2010). In this study, we searched for possible targets of silybin from PubChem database and obtained 26 active targets. And the results of target-based screening by Subpathway-GM indicated that ALDH1A1 was the potential target for Silybin in the treatment of prostate cancer and it could regulate the synthesis of all trans-retinoic acid as well as 9-cis-Retinoate which could inhibit the cancer cell-stimulated proliferation and differentiation of the pro-tumoral macrophages and to act as a tumor suppressor gene in the prostate cancer 


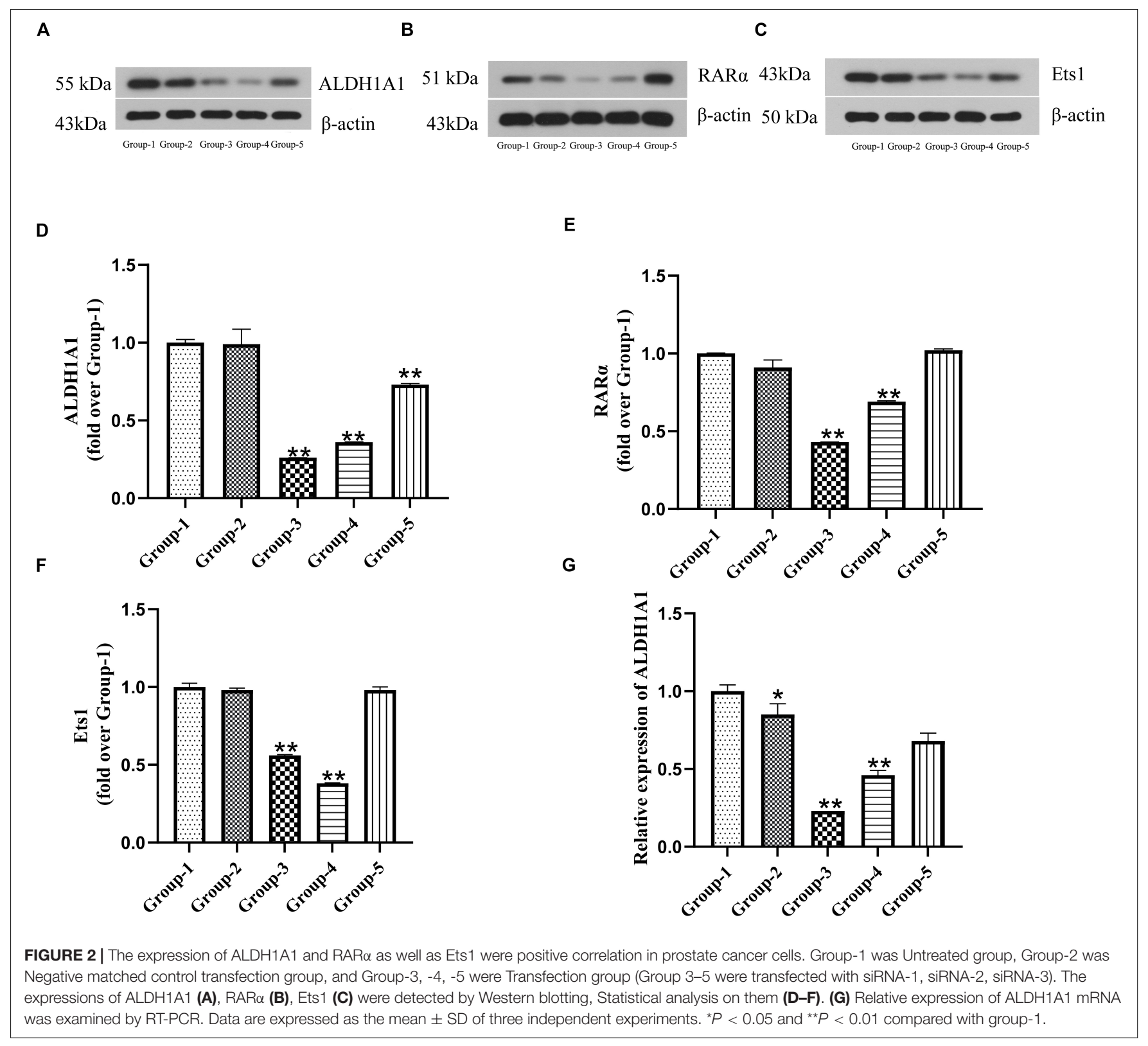

(Jiang et al., 2006; Tsagozis et al., 2014). Hellsten et al. (2011) proved that ALDH1A1 $(+)$ prostate cancer cells showed cancer stem cell-like characteristics such as increased self-renewing and colony forming capacity and tumorigenicity. Therefore, we successfully isolated the ALDH1A1(+) cells from DU145 cells by using magnetic bead cell sorting (MACS) to investigate the biological function. And results showed that ALDH1A1 promoted the proliferation, migration and invasion ability of prostate stem cancer cells which indicated that ALDH1A1 was indeed a potential biomarker for prostate stem cancer cells.

Actually, ALDH1A1 as the major enzyme for retinoic acid synthesis, is responsible for converting aldehyde to acid (Landrier et al., 2017). Some research showed that ALDH1A1 could transform precursor retinaldehydes into retinoic acid receptor (RAR) which was a kind of steroid hormone receptor
(Kiefer et al., 2012). Interestingly, deficiency of the ALDH1A1 gene also limited the formation of RAR (Shen et al., 2018). Besides, Hammond et al. (2001) demonstrated that antagonists of retinoic acid receptors (RARs) effectively inhibited the growth prostate carcinoma cells. In this study, we found that the

TABLE 2 | PCR primer sequence.

\begin{tabular}{lc}
\hline Gene & Primer sequence \\
\hline ALDH1A1 F & GGCAGCCATTCTTCTCA \\
ALDH1A1 R & TGTCCAAGTCGGCATCAG \\
$\beta$-actin F & ACCCTGAAGTACCCCATCGA \\
$\beta$-actin $R$ & CAAACATGATCTGGGTCATCT
\end{tabular}




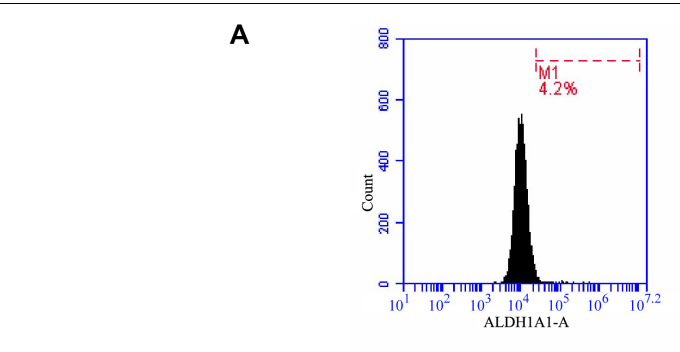

B

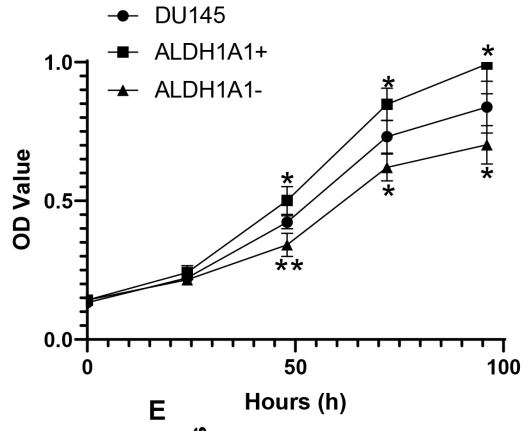

E

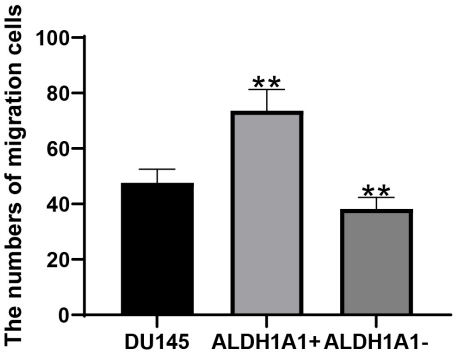

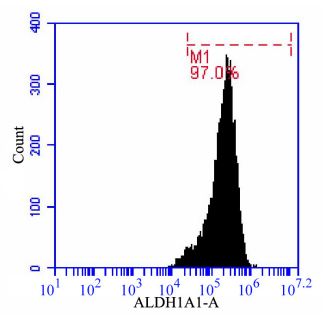

ALDH1A1+

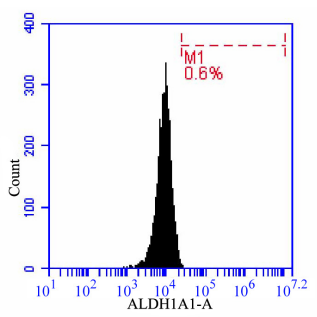

ALDH1A1-

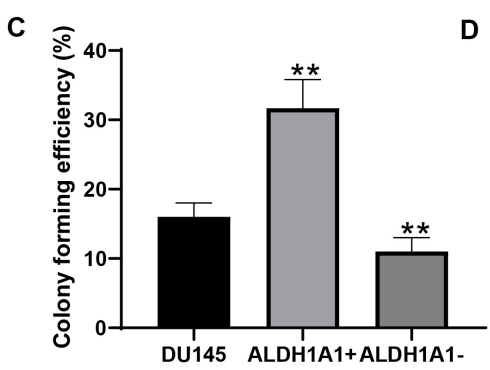

D $\overleftarrow{5}$

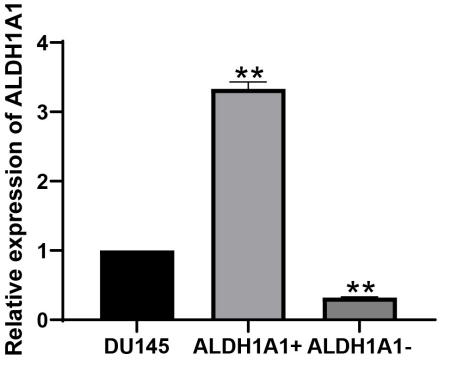

$\mathbf{F}$

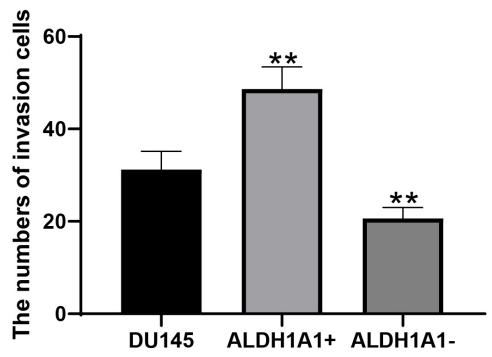

G

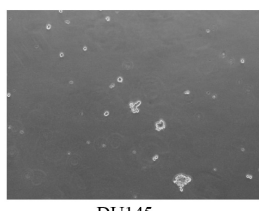

DU145

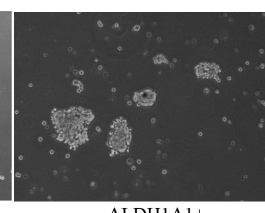

ALDH1A1+

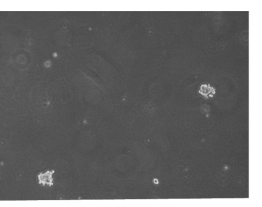

ALDH1A1-

I

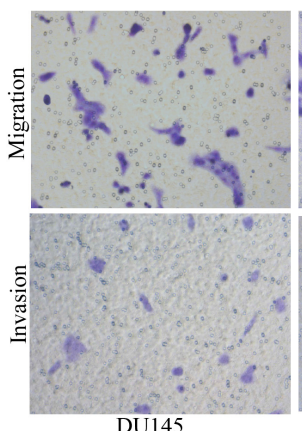

DU145

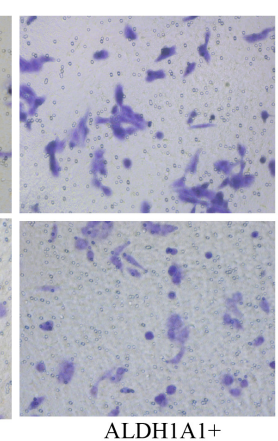

ALDH1A1+

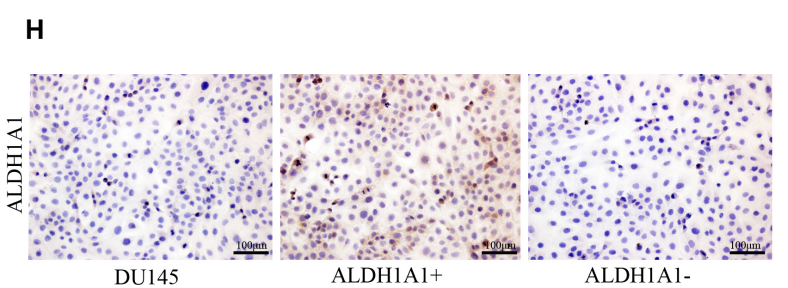

J

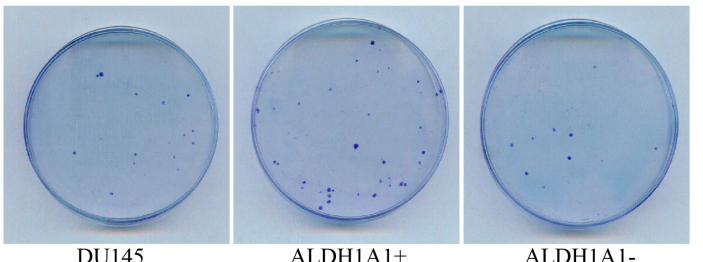

DU145

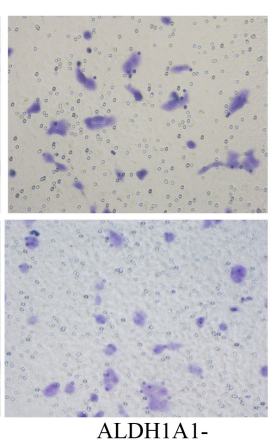

ALDH1A1-

FIGURE 3 | Screening and identification of ALDH1A1+ prostate cancer stem cell-like cells and their biological functions in vitro. Flow cytometry detected the expression of ALDH1A1 positive cells (A). The cells proliferative capacity was detected at 0, 24, 48, $72,96 \mathrm{~h}$ by MTT assay (B). Plating efficiency was tested by plate clonality assay (C), and was colony forming efficiency was calculated (F). The expression of ALDH1A1 was detected by Immunocytochemistry (H, 200×, brown yellow granules indicate positive reaction). miR-ALDH1A1 was detected by Rt-PCR (D). The images of Transwell assay were recorded (I) which revealed the numbers of migration (E) and invasion (F) cells. To detected assess the presence and self-renewal ability of cells by sphere-formation Assay (G). The expression of ALDH1A1 were detected with immunohistochemistry $\mathbf{( H )}$. Plate clonality was used to detect the colony ability of DU145 cells $(\mathbf{J})$. Data are expressed as the mean $\pm \mathrm{SD}$ of three independent experiments. ${ }^{\star} P<0.05$ and ${ }^{\star \star} P<0.01$ compared with DU145. 
A

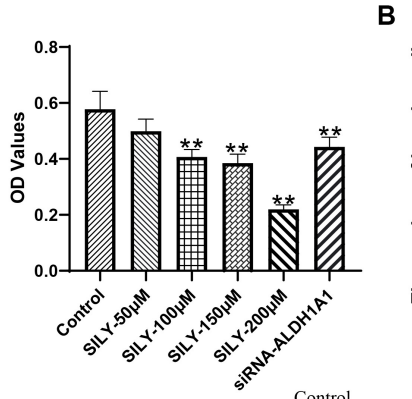

D

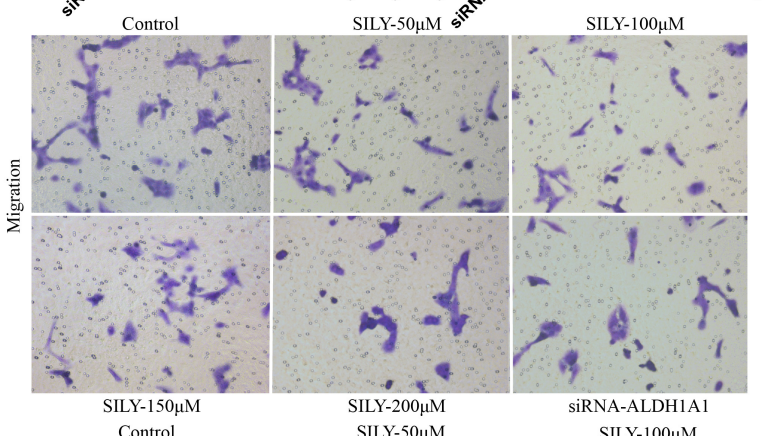

E

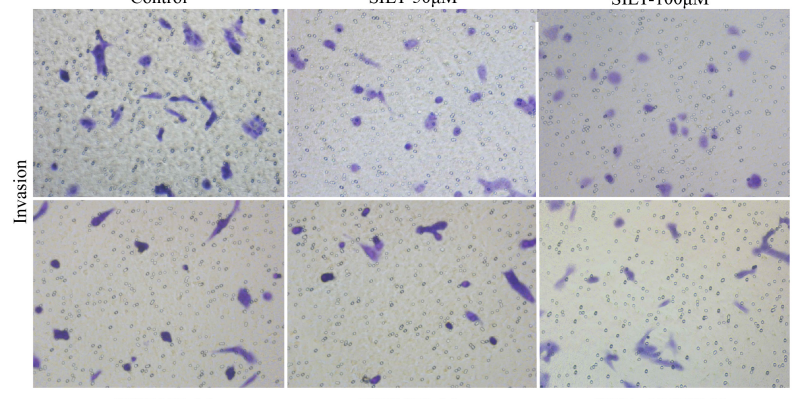

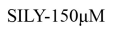

$\mathbf{F}$

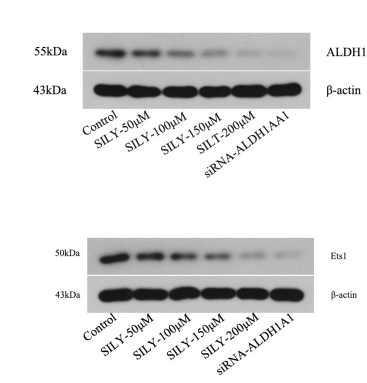

K

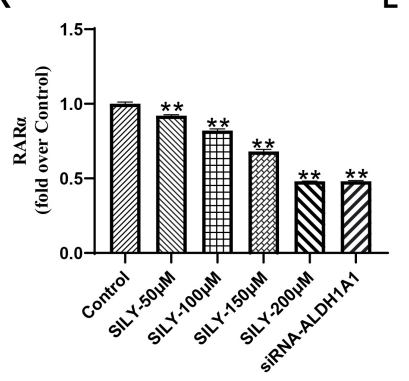

$\mathbf{L}$
G
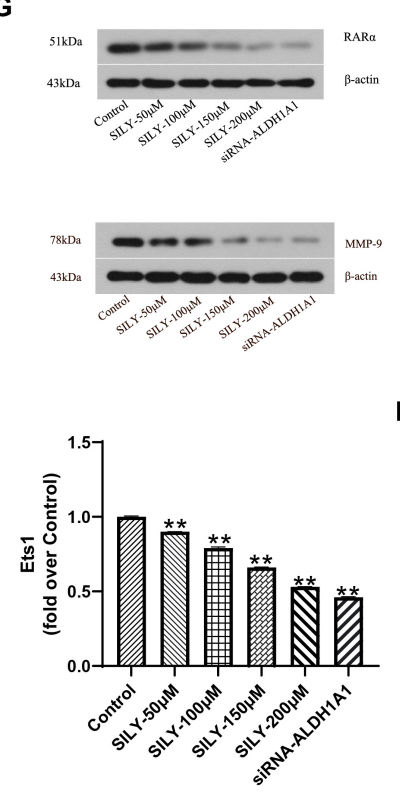

C

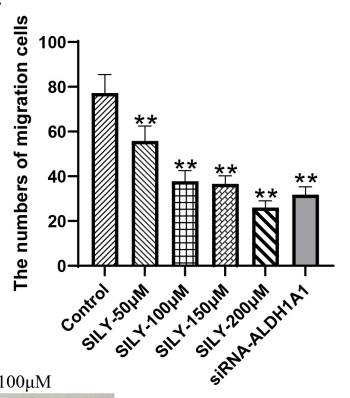

iRNA-ALDH1Al

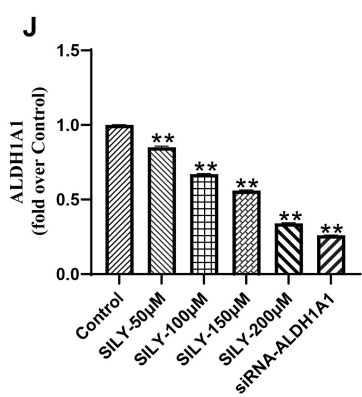

M

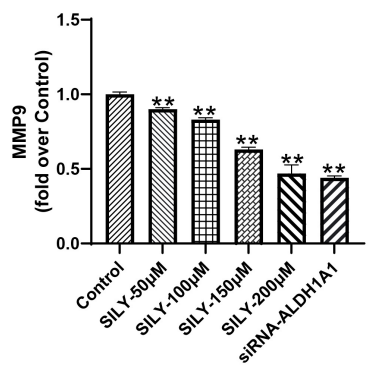

FIGURE 4 | Biological function of Silybin on DU145 cells. The cells proliferative capacity was assessed by MTT assay (A). The images of Transwell assay were recorded (D,E) which revealed the numbers of invasion (B) and migration (C) cells. The expressions of ALDH1A1 (F), RAR $\alpha(\mathbf{G})$, Ets1 (H), MMP9 (I) were detected by Western blotting, Statistical analysis on them (J-M). Data are expressed as the mean \pm SD of three independent experiments. ${ }^{*} P<0.05$ and ${ }^{* *} P<0.01$ compared with Control group. 
A
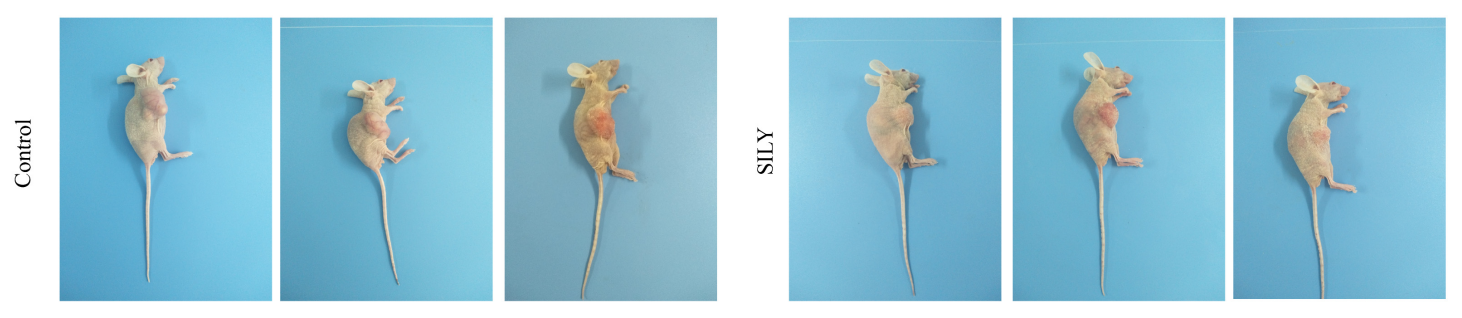

B
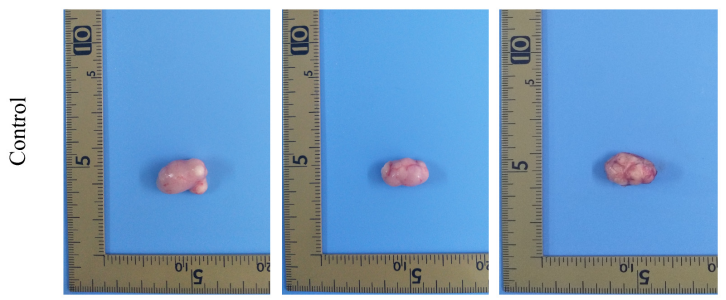

C

D
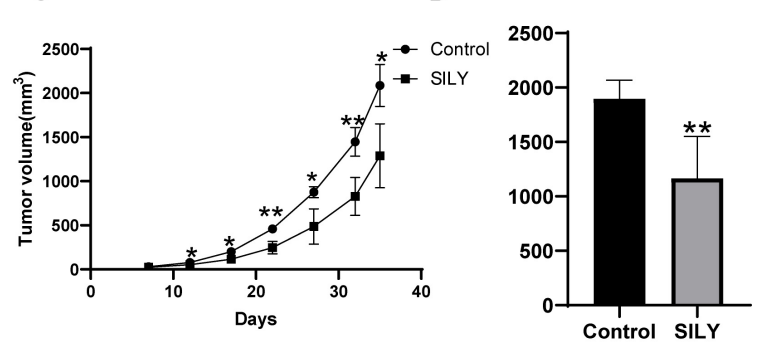

I

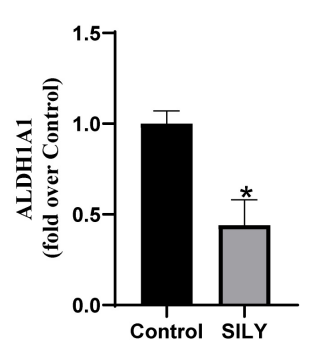

J

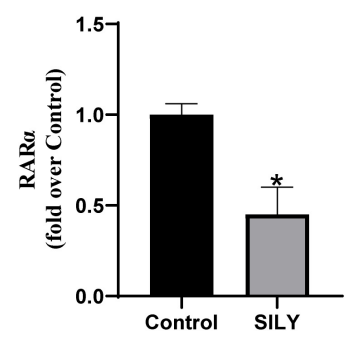

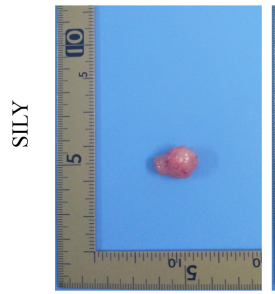

E

5Skba

$43 \mathrm{kaa}$

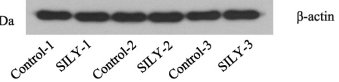

G

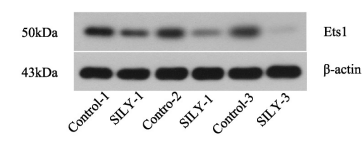

K

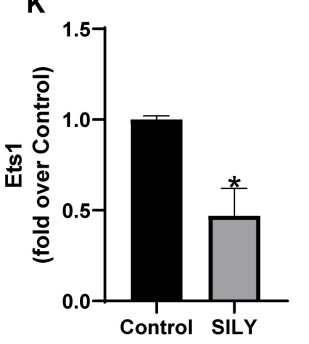

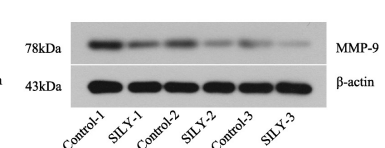
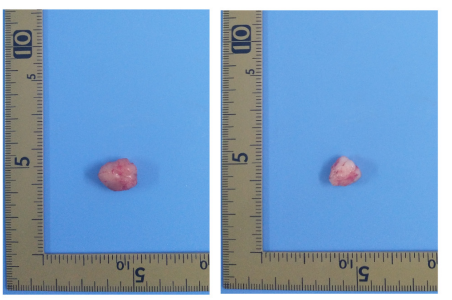

$\mathbf{F}$

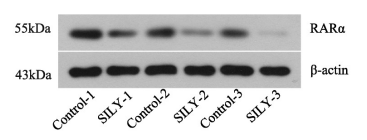

H

$\mathbf{L}$

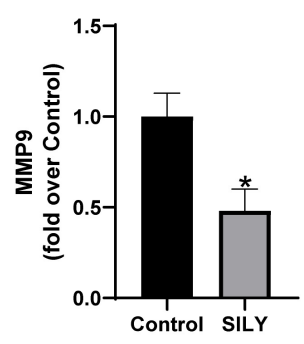

FIGURE $\mathbf{5}$ | Biological function of Silybin in vivo. The picture of nude mice (A) and tumor (B) as well as the tumor weight (D) were recorded after sacrifice. And the tumor volume was detected after tumor formation in nude mice every 5 days (C). The expressions of ALDH1A1 (E), RAR $\alpha$ (F), Ets1 (G), MMP9 (H) were detected by Western blotting, Statistical analysis on them (I-L). Data are expressed as the mean \pm SD of three independent experiments. ${ }^{\star} P<0.05$ and ${ }^{\star \star} P<0.01$ compared with Control group.

expression of ALDH1A1 was obviously decreased along with the reduced levels of RAR $\alpha$ after DU145 cells were interference by siRNA which was consistent with other studies. Previously, it had been exhibited that RAR $\alpha$ could bind to E26 transformationspecific-1 (Ets1) promoter and induced the expression of Ets-1 mRNA and protein levels in cancer cells (Raouf et al., 2000; So and Crowe, 2000). And the overexpression of Ets1 were always associated with malignant biological features of prostate cancer (Li et al., 2012). Because Ets1 could affect extracellular matrix (ECM) degradation which was the premise of tumor cell metastasis (Cao et al., 2015). And Ets1 production also decreased after interference by siRNA. These results indicated that ALDH1A1 expression was positively correlated RAR $\alpha$ with Ets1 in prostate cancer. ALDH1A1 promoted invasion and metastasis of prostate cancer by activating the RAR $\alpha$, which further activates Ets1.

Cancer stem cell hypothesis suggested that there are a few cells with stem cell-like characteristics in tumor tissues, which are able to proliferate, self-renew, differentiate in multiple ways, and promote the infinite proliferation of tumors and even the formation of new tumors (Mackillop et al., 1983). It has been proven that Silybin could inhibited the secretion of angiogenic factors and targeting the responses of endothelial angiogenic factors (motility, proliferation, survival, and differentiation) 
effectively for the treatment of prostate cancer cells (Singh et al., 2008). Our observation also exhibited that Silybin could suppress the proliferation, migration and invasion ability of prostate stem cancer cells. Besides, there is substantial evidence that decreased tumor cell proliferation and increased tumor cell apoptosis in mice significantly alleviated tumor growth (Chen et al., 2016). Regarding to tumor growth by measurements of tumor weight and volume, we found that tumor volume and weight of mice treated with Silybin was obviously reduced. As mentioned before, ALDH1A1 expression was positively correlated RAR $\alpha$ with Ets1 and metalloproteinase 9 (MMP9) was one of the most important substance related to cancer invasion and metastasis (Nazir et al., 2019). It has been reported that inhibiting the expression of Ets1 gene could reduce proteins of the MMP9 secretion and significantly inhibit the invasion of PC3 cells (Kato et al., 2012). Yang et al. (2018) also indicated that the migration, invasion ability of PCa cells could be inhibited by downregulated the expression of MMP9. As expected, Silybin presented inhibition of ALDH1A1 expression and along with the lower expressions of RAR $\alpha$, Ets1 and MMP9. Therefore, these results consistently demonstrated Silybin had therapeutic effect on prostate cancer by downregulated the expression of ALDH1A1 in vivo and vitro.

In summary, firstly we screened out Silybin metabolic pathway was highly correlated with ALDH1A1. And then utilizing in vitro and in vivo experimental systems, we show that ALDH1A1 promoted invasion and metastasis of prostate cancer by activating the RAR $\alpha$, which further activated Ets1, MMP9 and Silybin inhibited the expression of ALDH1A1 in prostate cancer, thereby inhibiting the activation of RAR and preventing the activation of Ets 1 to inhibit the growth and invasion of prostate cancer.

\section{DATA AVAILABILITY STATEMENT}

The raw data supporting the conclusions of this article will be made available by the authors, without undue reservation.

\section{REFERENCES}

Ahmad, A. E., Leao, R., and Hamilton, R. J. (2017). Radical prostatectomy for patients with oligometastatic prostate Cancer. Oncology (Williston Park) 31, 794-802.

Arcangeli, S., Pinzi, V., and Arcangeli, G. (2012). Epidemiology of prostate cancer and treatment remarks. World J. Radiol. 4, 241-246. doi: 10.4329/wjr.v4.i6.241

Cao, L., Xie, B., Yang, X., Liang, H., Jiang, X., Zhang, D., et al. (2015). MiR-324$5 \mathrm{p}$ suppresses hepatocellular carcinoma cell invasion by counteracting ECM degradation through post-transcriptionally downregulating ETS1 and SP1. PLoS One 10:e0133074. doi: 10.1371/journal.pone. 0133074

Chen, X., Li, C., He, T., Mao, J., Li, C., Lyu, J., et al. (2016). Metformin inhibits prostate cancer cell proliferation, migration, and tumor growth through upregulation of PEDF expression. Cancer Biol. Ther. 17, 507-514. doi: 10.1080/ 15384047.2016.1156273

Cheng, L., Qi, C., Zhuang, H., Fu, T., and Zhang, X. (2020). gutMDisorder: a comprehensive database for dysbiosis of the gut microbiota in disorders and interventions. Nucleic Acids Res. 48:7603.

\section{ETHICS STATEMENT}

The animal study was reviewed and approved by Institutional Animal Care and Use Committee (IACUC) of Heilongjiang University of Traditional Chinese Medicine.

\section{AUTHOR CONTRIBUTIONS}

YJ, JL, and DW conceived and designed the study. YJ, HS, and LJ performed the experiment. JL and DW guided the experimental operation. YJ, YQ, and DY drafted the manuscript. All the authors read and agreed to the manuscript.

\section{FUNDING}

This work was supported in part by the National Natural Science Foundation of China (grant No. 81804158), the Natural Science Foundation of Heilongjiang Province (grant No. H2016074), the China Postdoctoral Science Foundation (grant No. 2016M591566), and the Postdoctoral Foundation of Heilongjiang Province (grant No. LBH-Z15211).

\section{ACKNOWLEDGMENTS}

YJ is a postdoctoral fellow in the traditional Chinese medicine research station of Heilongjiang University of Chinese Medicine. We thank the traditional Chinese medicine research station for providing the experimental platform.

\section{SUPPLEMENTARY MATERIAL}

The Supplementary Material for this article can be found online at: https://www.frontiersin.org/articles/10.3389/fcell.2020. 574394/full\#supplementary-material

Cheng, L., Zhao, H., Wang, P., Zhou, W., Luo, M., Li, T., et al. (2019). Computational methods for identifying similar diseases. Mol. Ther. Nucleic Acids 18, 590-604. doi: 10.1016/j.omtn.2019.09.019

Deep, G., and Agarwal, R. (2010). Antimetastatic efficacy of silibinin: molecular mechanisms and therapeutic potential against cancer. Cancer Metastasis. Rev. 29, 447-463. doi: 10.1007/s10555-010-9237-9230

Di, J., Zheng, B., Kong, Q., Jiang, Y., Liu, S., Yang, Y., et al. (2019). Prioritization of candidate cancer drugs based on a drug functional similarity network constructed by integrating pathway activities and drug activities. Mol. Oncol. 13, 2259-2277. doi: 10.1002/1878-0261.12564

Di Costanzo, A., and Angelico, R. (2019). Formulation strategies for enhancing the bioavailability of silymarin: the state of the art. Molecules 24:2155. doi: 10.3390/molecules24112155

Du, X. Q., Li, X. R., Li, W., Yan, Y. T., and Zhang, Y. P. (2018). Identification and analysis of cancer diagnosis using probabilistic classification vector machines with feature selection. Curr. Bioinform. 13, 625-632. doi: 10.2174/ 1574893612666170405125637

Feng, Y. M. (2019). Gene therapy on the road. Curr. Gene. Ther. 19:6. doi: 10.2174/ 1566523219999190426144513 
Foj, L., and Filella, X. (2019). Identification of potential miRNAs biomarkers for high-grade prostate cancer by integrated bioinformatics analysis. Pathol. Oncol. Res. 25, 1445-1456. doi: 10.1007/s12253-018-0508-503

Gamat, M., and McNeel, D. G. (2017). Androgen deprivation and immunotherapy for the treatment of prostate cancer. Endocr. Relat. Cancer 24, T297-T310. doi: 10.1530/ERC-17-0145

Gao, L., Zhang, L. J., Li, S. H., Wei, L. L., Luo, B., He, R. Q., et al. (2018). Role of miR-452-5p in the tumorigenesis of prostate cancer: a study based on the Cancer Genome Atl(TCGA), Gene Expression Omnibus (GEO), and bioinformatics analysis. Pathol. Res. Pract. 214, 732-749. doi: 10.1016/j.prp. 2018.03.002

Green, A. K., Corty, R. W., Wood, W. A., Meeneghan, M., Reeder-Hayes, K. E., Basch, E., et al. (2015). Comparative effectiveness of mitoxantrone plus prednisone versus prednisone alone in metastatic castrate-resistant prostate cancer after docetaxel failure. Oncologist 20, 516-522. doi: 10.1634/ theoncologist.2014-2432

Hammond, L. A., Van Krinks, C. H., Durham, J., Tomkins, S. E., Burnett, R. D., Jones, E. L., et al. (2001). Antagonists of retinoic acid receptors (RARs) are potent growth inhibitors of prostate carcinoma cells. Br. J. Cancer 85, 453-462. doi: 10.1054/bjoc.2001.1939

Han, J., Han, X., Kong, Q., and Cheng, L. (2020). psSubpathway: a software package for flexible identification of phenotype-specific subpathways in cancer progression. Bioinformatics 36, 2303-2305. doi: 10.1093/bioinformatics/btz894

Han, J., Liu, S., Jiang, Y., Xu, C., Zheng, B., Jiang, M., et al. (2018). Inference of patient-specific subpathway activities reveals a functional signature associated with the prognosis of patients with breast cancer. J. Cell Mol. Med. 22, 43044316. doi: $10.1111 / \mathrm{jcmm} .13720$

Hellsten, R., Johansson, M., Dahlman, A., Sterner, O., and Bjartell, A. (2011). Galiellalactone inhibits stem cell-like ALDH-positive prostate cancer cells. PLoS One 6:e22118. doi: 10.1371/journal.pone.0022118

Huang, H., Zhang, Q., Ye, C., Lv, J. M., Liu, X., Chen, L., et al. (2017). Identification of prognostic markers of high grade prostate cancer through an integrated bioinformatics approach. J. Cancer Res. Clin. Oncol. 143, 2571-2579. doi: 10. 1007/s00432-017-2497-2490

Jahanafrooz, Z., Motamed, N., Rinner, B., Mokhtarzadeh, A., and Baradaran, B. (2018). Silibinin to improve cancer therapeutic, as an apoptotic inducer, autophagy modulator, cell cycle inhibitor, and microRNAs regulator. Life Sci. 213, 236-247. doi: 10.1016/j.lfs.2018.10.009

Ji, J., Tang, J., Xia, K.-J., and Jiang, R. (2019). LncRNA in tumorigenesis microenvironment. Curr. Bioinform. 14, 640-641. doi: 10.2174/ 157489361407190917161654

Jiang, A. L., Zhang, P. J., Chen, W. W., Liu, W. W., Yu, C. X., Hu, X. Y., et al. (2006). Effects of 9-cis retinoic acid on human homeobox gene NKX3.1 expression in prostate cancer cell line LNCaP. Asian J. Androl. 8, 435-441. doi: 10.1111/j. 1745-7262.2006.00171.x

Kato, T., Fujita, Y., Nakane, K., Kojima, T., Nozawa, Y., Deguchi, T., et al. (2012). ETS1 promotes chemoresistance and invasion of paclitaxel-resistant, hormone-refractory PC3 prostate cancer cells by up-regulating MDR1 and MMP9 expression. Biochem. Biophys. Res. Commun. 417, 966-971. doi: 10. 1016/j.bbrc.2011.12.047

Kiefer, F. W., Vernochet, C., O’Brien, P., Spoerl, S., Brown, J. D., Nallamshetty, S., et al. (2012). Retinaldehyde dehydrogenase 1 regulates a thermogenic program in white adipose tissue. Nat. Med. 18, 918-925. doi: 10.1038/nm.2757

Kimura, T., and Egawa, S. (2018). Epidemiology of prostate cancer in Asian countries. Int. J. Urol. 25, 524-531. doi: 10.1111/iju.13593

Lai, H. Y., Feng, C. Q., Zhang, Z. Y., Tang, H., Chen, W., and Lin, H. (2018). A brief survey of machine learning application in cancerlectin identification. Curr. Gene. Ther. 18, 257-267. doi: 10.2174/1566523218666180913112751

Landrier, J. F., Kasiri, E., Karkeni, E., Mihaly, J., Beke, G., Weiss, K., et al. (2017). Reduced adiponectin expression after high-fat diet is associated with selective up-regulation of ALDH1A1 and further retinoic acid receptor signaling in adipose tissue. FASEB J. 31, 203-211. doi: 10.1096/fj.201600263RR

Lawrence, M. G., Obinata, D., Sandhu, S., Selth, L. A., Wong, S. Q., Porter, L. H., et al. (2018). Patient-derived models of abiraterone- and enzalutamide-resistant prostate cancer reveal sensitivity to ribosome-directed therapy. Eur. Urol. 74, 562-572. doi: 10.1016/j.eururo.2018.06.020

Li, B., Shimizu, Y., Kobayashi, T., Terada, N., Yoshimura, K., Kamba, T., et al. (2012). Overexpression of ETS-1 is associated with malignant biological features of prostate cancer. Asian J. Androl. 14, 860-863. doi: 10.1038/aja. 2012.107

Li, C., Han, J., Yao, Q., Zou, C., Xu, Y., Zhang, C., et al. (2013). Subpathway-GM: identification of metabolic subpathways via joint power of interesting genes and metabolites and their topologies within pathways. Nucleic Acids Res. 41:e101. doi: 10.1093/nar/gkt161

Li, T., Su, Y., Mei, Y., Leng, Q., Leng, B., Liu, Z., et al. (2010). ALDH1A1 is a marker for malignant prostate stem cells and predictor of prostate cancer patients' outcome. Lab. Invest. 90, 234-244. doi: 10.1038/labinvest. 2009.127

Liao, Z. J., Li, D. P., Wang, X. R., Li, L. S., and Zou, Q. (2018). Cancer diagnosis through IsomiR expression with machine learning method. Curr. Bioinform. 13, 57-63. doi: 10.2174/1574893611666160609081155

Lin, M., Li, X., Guo, H., Ji, F., Ye, L., Ma, X., et al. (2019). Identification of bone metastasis-associated genes of gastric cancer by genome-wide transcriptional profiling. Curr. Bioinform. 14, 62-69. doi: 10.2174/ 1574893612666171121154017

Liu, H., Luo, L. B., Cheng, Z. Z., Sun, J. J., Guan, J. H., Zheng, J., et al. (2018). Group-sparse modeling drug-kinase networks for predicting combinatorial drug sensitivity in cancer cells. Curr. Bioinform. 13, 437-443. doi: 10.2174/ 1574893613666180118104250

Mackillop, W. J., Ciampi, A., Till, J. E., and Buick, R. N. (1983). A stem cell model of human tumor growth: implications for tumor cell clonogenic assays. J. Natl. Cancer Inst. 70, 9-16.

Nazir, S. U., Kumar, R., Singh, A., Khan, A., Tanwar, P., Tripathi, R., et al. (2019). Breast cancer invasion and progression by MMP-9 through Ets- 1 transcription factor. Gene 711:143952. doi: 10.1016/j.gene.2019.143952

Qu, K. Y., Gao, F., Guo, F., and Zou, Q. (2019). Taxonomy dimension reduction for colorectal cancer prediction. Comput. Biol. Chem. 83:107160. doi: 10.1016/j. compbiolchem.2019.107160

Raouf, A., Li, V., Kola, I., Watson, D. K., and Seth, A. (2000). The Ets1 protooncogene is upregulated by retinoic acid: characterization of a functional retinoic acid response element in the Ets1 promoter. Oncogene 19, 1969-1974. doi: 10.1038/sj.onc. 1203505

Shen, Q., Yasmeen, R., Marbourg, J., Xu, L., Yu, L., Fadda, P., et al. (2018). Induction of innervation by encapsulated adipocytes with engineered vitamin a metabolism. Transl. Res. 192, 1-14. doi: 10.1016/j.trsl.2017.10.005

Singh, R. P., Raina, K., Sharma, G., and Agarwal, R. (2008). Silibinin inhibits established prostate tumor growth, progression, invasion, and metastasis and suppresses tumor angiogenesis and epithelial-mesenchymal transition in transgenic adenocarcinoma of the mouse prostate model mice. Clin. Cancer Res. 14, 7773-7780. doi: 10.1158/1078-0432.CCR-08-1309

So, E. N., and Crowe, D. L. (2000). Characterization of a retinoic acid responsive element in the human ets-1 promoter. IUBMB Life 50, 365-370. doi: 10.1080/ 713803742

Su, R., Liu, X., Wei, L., and Zou, Q. (2019). Deep-Resp-forest: a deep forest model to predict anti-cancer drug response. Methods 166, 91-102. doi: 10.1016/j.ymeth. 2019.02.009

Sun, J., Li, S., Wang, F., Fan, C., and Wang, J. (2019). Identification of key pathways and genes in PTEN mutation prostate cancer by bioinformatics analysis. BMC Med. Genet. 20:191. doi: 10.1186/s12881-019-0923-927

Tang, W., Wan, S., Yang, Z., Teschendorff, A. E., and Zou, Q. (2018). Tumor origin detection with tissue-specific miRNA and DNA methylation markers. Bioinformatics 34, 398-406. doi: 10.1093/bioinformatics/btx622

Ting, H., Deep, G., and Agarwal, R. (2013). Molecular mechanisms of silibininmediated cancer chemoprevention with major emphasis on prostate cancer. AAPS J. 15, 707-716. doi: 10.1208/s12248-013-9486-9482

Tsagozis, P., Augsten, M., and Pisa, P. (2014). All trans-retinoic acid abrogates the pro-tumorigenic phenotype of prostate cancer tumor-associated macrophages. Int. Immunopharmacol. 23, 8-13. doi: 10.1016/j.intimp.2014.07.037

Varghese, L., Agarwal, C., Tyagi, A., Singh, R. P., and Agarwal, R. (2005). Silibinin efficacy against human hepatocellular carcinoma. Clin. Cancer Res. 11, 84418448. doi: 10.1158/1078-0432.CCR-05-1646

Wu, Y., Lu, X., Shen, B., and Zeng, Y. (2019). The Therapeutic Potential and Role of miRNA, IncRNA, and circRNA in Osteoarthritis. Curr. Gene. Ther. 19, 255-263. doi: 10.2174/1566523219666190716092203

Yang, F., Yu, N., Wang, H., Zhang, C., Zhang, Z., Li, Y., et al. (2018). Downregulated expression of hepatoma-derived growth factor inhibits migration and invasion 
of prostate cancer cells by suppressing epithelial-mesenchymal transition and MMP2. MMP9. PLoS One 13:e0190725. doi: 10.1371/journal.pone. 0190725

Yoshida, A., Hsu, L. C., and Dave, V. (1992). Retinal oxidation activity and biological role of human cytosolic aldehyde dehydrogenase. Enzyme 46, 239244. doi: $10.1159 / 000468794$

Zeng, W., Wang, F., Ma, Y., Liang, X. C., and Chen, P. (2019). Dysfunctional mechanism of liver cancer mediated by transcription factor and non-coding RNA. Curr. Bioinform. 14, 100-107. doi: $10.2174 / 1574893614666181119121916$

Zhang, Z. M., Tan, J. X., Wang, F., Dao, F. Y., Zhang, Z. Y., and Lin, H. (2020). Early diagnosis of hepatocellular carcinoma using machine learning method. Front. Bioeng. Biotechnol. 8:254. doi: 10.3389/fbioe.2020. 00254
Zhou, L. Y., Qin, Z., Zhu, Y. H., He, Z. Y., and Xu, T. (2019). Current RNAbased therapeutics in clinical trials. Curr. Gene. Ther. 19, 172-196. doi: 10.2174/ 1566523219666190719100526

Conflict of Interest: The authors declare that the research was conducted in the absence of any commercial or financial relationships that could be construed as a potential conflict of interest.

Copyright $\odot 2020$ Jiang, Song, Jiang, Qiao, Yang, Wang and Li. This is an open-access article distributed under the terms of the Creative Commons Attribution License (CC BY). The use, distribution or reproduction in other forums is permitted, provided the original author(s) and the copyright owner(s) are credited and that the original publication in this journal is cited, in accordance with accepted academic practice. No use, distribution or reproduction is permitted which does not comply with these terms. 